\section{Position Paper on Kawasaki Disease in India: Pertinent Issues}

We read with interest the recently published IAP position paper on Kawasaki disease (KD) [1]. We would like to highlight the following issues that require further consideration.

Under laboratory investigations, it is noted that serum levels of NT-pro-BNP (N-terminal pro-brain natriuretic peptide $)>225 \mathrm{pg} / \mathrm{mL}$ can assist in the diagnosis of $\mathrm{KD}(86.5 \%$ sensitivity and $94.8 \%$ specificity to suggest myocardial dysfunction). However, in the subsequent section, authors mention that cut-off values for NT-Pro-BNP indicative of myocardial involvement are yet to be clearly defined. The AHA statement [2] also states that this biomarker may not have sufficient discriminative ability. It is notable that during childhood, NTPro-BNP is known to vary with age and therefore, it has been suggested that a single cut-off value based on ROC analysis would be inappropriate $[3,4]$.

It is mentioned that it may take 36-48 hours for the fever to subside in IVIG-responsive patients [1]. However, both this position paper and the AHA statement define IVIG resistance as persistence or recurrence of fever 36 hours after the end of IVIG infusion. Several recent studies and the Japanese Society of Pediatric Cardiology and Cardiac Surgery guidelines suggest a 48-hour time frame for the same [5]. The 36-hour cut-off, when applied strictly, could potentially lead to over-diagnosis of IVIG resistance. This is a pertinent issue that needs further exploration, considering that the time taken for IVIG infusion itself can be variable (typically 12 hours in North America and 20-24 hours in Japan) [5]. AHA recommends IVIG infusion over 10-12 hours (as opposed to 12-24 hours recommended by the authors) [1,2].

There are certain variations in the definition of recurrence. Recurrent $\mathrm{KD}$ is defined as a repeat episode of $\mathrm{KD}$ after complete resolution of the first episode [1,2]. Acute illness in $\mathrm{KD}$ usually lasts for 4 to 6 weeks and several Japanese surveys have classified $\mathrm{KD}$ as recurrent if there is an interval of at least two months from the onset of the first illness to onset of the new episode [6].

In the paper, the available Indian data has not been critically evaluated. It is imperative to consider relevant local data to bring in the much needed Indian perspective. In the process, lack of good quality data on the disease epidemiology and the importance of a national registry could have been highlighted. Finally, a conflict of interest statement by the authors is missing.

Gowda Parameshwara Prashanth ${ }^{1 *}$ and Anita TANDON ${ }^{2}$ Departments of Pediatrics, ${ }^{1}$ College of Medicine and Health Sciences,

\section{REFERENCES}

\author{
National University of Science and Technology, \\ Muscat; and ${ }^{2}$ Sohar Regional Teaching Hospital, \\ Sohar; Sultanate of Oman. \\ *prashanth_lucknow@rediffmail.com
}

1. Shenoy B, Singh S, Ahmed MZ, et al. Indian Academy of Pediatrics Position Paper on Kawasaki Disease. Indian Pediatr. 2020;57:1040-48.

2. McCrindle BW, Rowley AH, Newburger JW, et al. Diagnosis, Treatment, and Long-Term Management of Kawasaki Disease: A Scientific Statement for Health Professionals from the American Heart Association. Circulation. 2017; 135:e927-99.

3. Albers S, Mir TS, Haddad M, Läer S. N-terminal pro brain natriuretic peptide: Evaluation of pediatric reference values including method comparison and inter-laboratory variability. Clin Chem Lab Med. 2006;44:80-85.

4. Dahdah N, Fournier A. Natriuretic peptides in Kawasaki disease: The myocardial perspective. Diagnostics (Basel). 2013; 3:1-12.

5. Research Committee of the Japanese Society of Pediatric Cardiology; Cardiac Surgery Committee for Development of Guidelines for Medical Treatment of Acute Kawasaki Disease. Guidelines for medical treatment of acute Kawasaki disease: Report of the Research Committee of the Japanese Society of Pediatric Cardiology and Cardiac Surgery (2012 revised version). Pediatr Int. 2014;56:135-58. Erratum in: Pediatr Int. 2016;58:675.

6. Hirata S, Nakamura Y, Yanagawa $\mathrm{H}$. Incidence rate of recurrent Kawasaki disease and related risk factors: From the results of nationwide surveys of Kawasaki disease in Japan. Acta Paediatr. 2001;90:40-4.

\section{AUTHORS'REPLY}

We are in agreement with the author that NT-pro-BNP is not a well established tool for the diagnosis of KD. As rightly pointed out, NT-pro-BNP varies with age and the values provided in the paper are from the study by Dahdah, et al. [1]. It must be said that one should refer to age related upper limits of normal and it is also useful to keep in mind to avoid making diagnosis of Kawasaki disease just on the basis of NT-pro-BNP alone. Though there has been a global effort to identify a suitable biomarker for KD diagnosis, but that still remains elusive. NT-pro-BNP is presently an accessible tool in many centers and the facts relating to this tool has been added as an addendum in the paper.

Regarding the 36 hours (post intravenous immunoglobulin infusion) being the cut-off for the diagnosis of IVIg resistance, it was more of an adaptation from the American Heart Association (AHA) guidelines [2]. It is important to keep in mind that this period is after the completion of IVIg infusion and the duration of the IVIg infusion (10-12 hours vs 12-24 hours) does not matter much. The longer infusion period would specially apply to the context of school-going children with the disease when a higher total dose of IVIg needs to be infused. It needs to be emphasized that in a disease like $\mathrm{KD}$, it might be useful to 
overtreat rather than undertreat to prevent lifelong complications due to coronary aneurysms.

The definition of recurrent KD would essentially mean a recurrence after documented remission of the first episode of KD (clinically, echocardiography and laboratory). It goes without saying that this period would be at least for about 4 to 6 weeks.

This is a position paper on KD providing diagnostic and therapeutic guidelines for practising pediatricians across the country. We did not intend to highlight or analyze Indian data. Moreover, data on KD in India is predominantly emerging from few centres and not representative of the scenario in the whole country.

BHASKAR SHENOY Department of Pediatrics, Manipal Hospitals, Bangalore, Karnataka, India. bshenoy@gmail.com

\section{Inclusion of Multisystem Inflammatory Syndrome in Children and Adolescents Temporally Related to COVID -19 in the Differential Diagnosis of Kawasaki Disease}

It was an interesting and informative read to go through the IAP position paper on Kawasaki disease (KD) published recently [1]. It was indeed necessary to have a nation-wide consensus, which is suitable for India where the distribution of health resources is unequal and constrained. KD has become one of the leading causes of acquired heart disease in many developed countries of the world [1]. With almost a year of coronavirus disease 19 (COVID-19) pandemic, a new hyper-inflammatory syndrome affecting children has been observed, and variously labeled as Multisystem inflammatory syndrome in children (MIS-C) associated with COVID-19 or Pediatric inflammatory multisystem syndrome-temporally associated with SARS-CoV-2 (PIMS-TS) [2,3]. This condition has clinical features which overlap with other inflammatory diseases in childhood like Kawasaki disease (KD) and toxic shock syndrome (TSS) [2].

The position paper [1] mentions various differential diagnosis of KD, but does not mention PIMS-TS or MIS-C. It is important that this entity should be considered in the differential diagnosis of $\mathrm{KD}$, as the clinical presentation is very much overlapping, though the underlying mechanism for hyperinflammation is different. In KD inflammation of the coronary arteries is due to IL-1, the myocardial dysfunction and higher severity of the 2019-nCoV infection is predominantly driven by IL-6 and IL-10 in MIS-C [4]. Three different phenotypes of hyperinflammation in children has been speculated as classic KD, PIMS-TS and macrophage activation syndrome [4]. Though IVIG and steroids are the mainstay of therapy in these conditions and aspirin also important in $\mathrm{KD}$, the prognosis and long term follow up are different. Hence the authors would like to suggest that this evolving inflammatory disease should be included in the position paper as one of the differentials for KD.

Tushar Jagzape* ANd ANIL Kumar Goel Department of Pediatrics, AIIMS, Raipur, Chattisgarh, India. *tusharjagzape@aiimsraipur.edu.in

\section{REFERENCES}

1. Shenoy B, Singh S, Ahmed MZ, Pal P, Balan S, Vishwanthan V. Indian Academy of Pediatrics Position Paper on Kawasaki Disease. Indian Pediatr. 2020;57: 1040-48.

2. Royal College of Pediatrics and Child Health. Guidance-Pediatric multisystem inflammatory syndrome temporally associated with COVID-19, 2020. Accessed December 02, 2020. Available from: https://www.rcpch.ac.uk/resources/ guidance-pediatric-multi system-inflammatory-syndrometemporally-associated covid-19

3. Bhat CS, Gupta L, Balasubramanians, Singh S, Ramanan AV. Hyperinflammatory syndrome in children associate with COVID-19: Need for awareness. Indian Pediatr. 2020;57:92935 .

4. Shulman ST. Pediatric coronavirus disease-2019-associated multisystem inflammatory syndrome. J Pediatric Infect Dis. 2020;9:285-6.

\section{AUTHORS'REPLY}

We thank the authors for raising this pertinent point. We are in total agreement that MISC (PIMS) is one of the differential diagnoses of Kawasaki disease. The draft of this article; however, was prepared prior to the onset of COVID-19 pandemic, and thus it was missed out in the list of differential diagnosis of KD in the consensus statement. The committee has modified the differential diagnosis as follows:

\section{Differential Diagnosis of Kawasaki Disease}

1. Infections - Bacterial (streptococcal, leptospirosis, rickettsia), Viral (measles, adenovirus, Epstein Barr virus).

2. Toxin related-Staphylococcal scalded skin syndrome, toxic epidermal necrolysis

3. Inflammatory-Systemic juvenile idiopathic arthritis

4. Drug hypersensitivity - Steven-Johnson syndrome, drug reaction with eosinophilia and systemic symptoms (DRESS), mercury hypersensitivity.

5. Multisystem inflammatory disease of childhood temporally related to COVID-19 (MISC-C): A condition recognized and described during the COVID-19 pandemic. This occurs largely in children, usually above 5 years of age, as a short term illness with high grade fever and often with shock with multisystem inflammation. One of the phenotypes can 